\title{
Imaging techniques for the diagnosis of pulmonary embolism
}

\author{
This article was published in the following Dove Press journal: \\ Reports in Medical Imaging \\ 10 December 2010 \\ Number of times this article has been viewed
}

\section{Donia Aghajanzadeh' \\ Natig Gassanov' \\ Matthias Schmidt ${ }^{2}$ \\ Nasser Semmo ${ }^{3}$ \\ Fikret Er'}

'Department of Internal Medicine III, ${ }^{2}$ Department of Nuclear Medicine, University of Cologne, Cologne,

Germany; ${ }^{3}$ Department of Medicine II, University Hospital Freiburg, Freiburg, Germany
Correspondence: Fikret Er

Department of Internal Medicine III, University of Cologne, 62, Kerpener Str, Cologne 50924, Germany

Tel +49 22I 47832544

Fax +49 22I 478 327। 2

Email fikret.er@uk-koeln.de
Abstract: Pulmonary embolism (PE) is a cardiovascular emergency with high morbidity and mortality. The diagnostic workup of patients with suspected acute PE remains difficult due to a wide spectrum of clinical presentations. There is still no diagnostic test that is accurate, safe, readily available, and cost-effective. Pulmonary angiography has a high diagnostic accuracy, but it is an invasive and resource-demanding procedure. Noninvasive imaging tests including computerized tomographic pulmonary angiography and ventilation/perfusion scanning are well validated for the diagnosis of PE, but have limited sensitivity and specificity. For optimal efficiency, the choice of the initial imaging modality should be guided by the clinical probability assessment and D-dimer testing. This review covers the performance of different diagnostic tests and presents a diagnostic algorithm for PE diagnosis.

Keywords: pulmonary embolism, diagnosis, algorithm, imaging, cardiovascular, mortality

\section{Introduction}

Acute pulmonary embolism (PE) is one of the most common cardiovascular emergencies and is the main cause of death in hospitalized patients older than 65 years. ${ }^{1}$ The annual incidence of $\mathrm{PE}$ has been reported to be around 1/500 persons, but the true incidence is likely to be larger due to nonspecific clinical presentations of PE. ${ }^{2-4}$ Acute PE spans a wide clinical spectrum with largely nonspecific signs and symptoms. Therefore, the diagnostic workup of patients with clinically suspected PE is challenging and remains a major clinical problem. ${ }^{5}$ Since early initiation of antithrombotic therapy in patients with proven PE highly improves survival, immediate and accurate diagnostic tests are of great clinical relevance. ${ }^{6}$ The optimal diagnostic imaging modality for the diagnosis of PE continues to be debated. There are many types of imaging techniques that have found an application in patients with clinical suspicion of PE, including computerized tomographic pulmonary angiography (CTPA), ventilation-perfusion (V/Q) scanning, conventional pulmonary arteriography, echocardiography, magnetic resonance imaging (MRI), and imaging for deep vein thrombosis as an origin for acute PE. At present, CTPA is used in the vast majority of patients with suspected PE. Conventional pulmonary angiography (PA) is still the reference standard for PE diagnosing. As imaging tests with a 100\% sensitivity and specificity for acute PE are not available, limitations can be better managed by combining imaging techniques, clinical probability assessment, and D-dimer testing.

This review focuses on currently available and validated imaging techniques for the diagnosis of PE. A straightforward diagnostic algorithm is then presented. Newer 
imaging techniques such as helical CT and MRI are not evaluated because of the limited data currently available.

\section{Imaging techniques Chest radiography}

Chest radiography in patients with PE is usually abnormal. Common radiographic findings include plate-like atelectasis, pleural effusion, pulmonary infiltrates, and elevation of a hemidiaphragm. ${ }^{7}$ However, these signs are nonspecific. Classic signs of pulmonary infarction such as Hampton's hump (wedge-shaped consolidation in the lung periphery) or Westermark's sign (local oligemia) are suggestive but infrequent. In general, chest radiography cannot be used to diagnose or exclude PE. As an initial diagnostic test, its main value is in the differential diagnosis of other cardiorespiratory diseases, which mimic the clinical presentation of PE, such as pneumonia, pneumothorax, rib fracture, or congestive heart failure.

The advantages are that chest radiography excludes other causes of dyspnea and chest pain, and the disadvantage is that encountered findings are nonspecific.

\section{Computerized tomographic pulmonary angiography}

CTPA is increasingly being used as the main thoracic imaging test for the evaluation of PE. The well-recognized advantages include speed, wide availability, ability to define associated or alternative diagnoses, direct visualization of the thrombus, and the high number of definitive diagnostic results (either positive or negative) (Figure 1). ${ }^{8}$ Data from several studies comparing the performance of the first-generation single-detector (SD) CTPA with pulmonary arteriography reported wide variations in both sensitivity $(53 \%-100 \%)$ and specificity $(81 \%-100 \%)$ of SD-CTPA. ${ }^{9}$ In two large prospective studies, SD-CTPA had an overall sensitivity for $\mathrm{PE}$ of around $70 \%$ and specificity of $90 \%$ and is, therefore, considered as too insensitive to be used as a single test for ruling out PE. ${ }^{10,11}$ Furthermore, the sensitivity proved to depend on the location of PE with a sensitivity of $86 \%$ for segmental or larger PE and merely $21 \%$ for subsegmental PE. ${ }^{11}$ Two large outcome studies stressed the importance of additional ultrasonography in case of a negative SD-CTPA. ${ }^{12,13}$ With the emergence of multidetector-CTPA (MD-CTPA), CTPA has gained substantially in scanning speed and resolution allowing decreased section thickness, reduced scanning times, and adequate visualization of pulmonary arteries up to segmental and subsegmental vessels. ${ }^{14}$ Comparing the diagnostic accuracy of MD-CTPA with that of PA, a sensitivity and specificity for PE of above $90 \%$ have

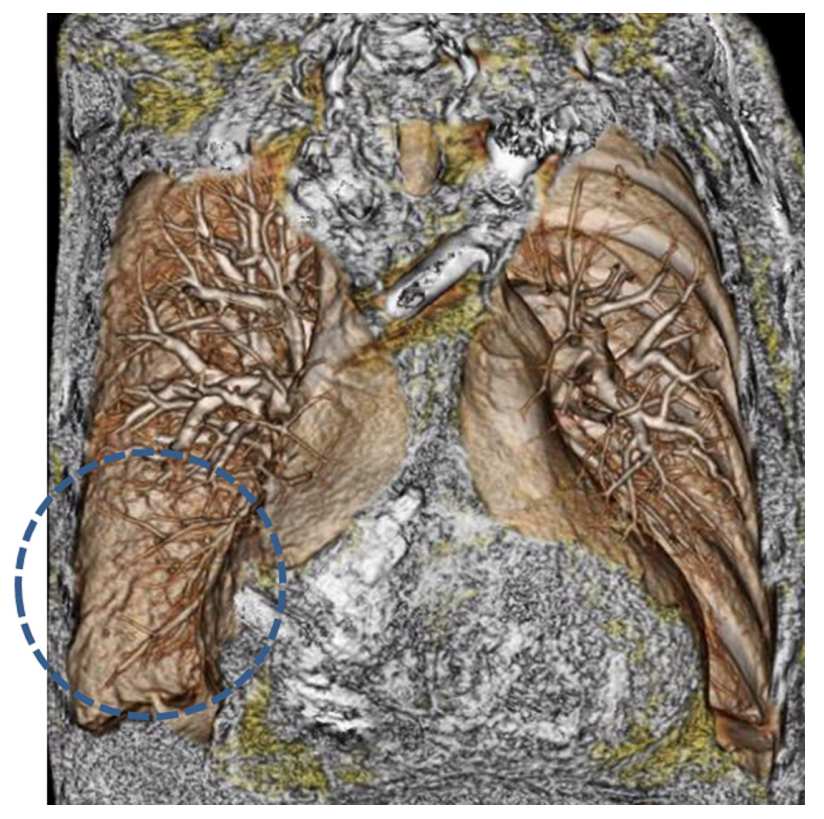

Figure I Diagnosis of PE with CT. 3D reconstruction of CT angiography reveals that the right inferior pulmonary artery with its side branches is almost completely absent due to thromboembolic occlusion. Dotted circle marks the area of hypoperfusion. Abbreviations: 3D, three-dimensional; PE, pulmonary embolism; CT, computed tomography.

been reported. ${ }^{15,16}$ However, the overall diagnostic sensitivity of MD-CTPA reported in the large multicenter Prospective Investigation of Pulmonary Embolism Diagnosis (PIOPED) II study was only $83 \%$ (95\% confidence interval, $76 \%-92 \%) .{ }^{17}$ This study emphasized the influence of objective clinical probability, as assessed by the Wells criteria (Table 1) to rule out PE. The predictive value of MD-CTPA was high with an $89 \%-95 \%$ negative predictive value (NPV) and a $92 \%-96 \%$ positive predictive value (PPV) in patients with a concordant clinical assessment, whereas it was lower (NPV 60\% and

Table I Wells rules for clinical risk stratification in patients with suspected PE

\begin{tabular}{ll}
\hline Symptom & Points \\
\hline Clinical signs and symptoms of deep venous thrombosis & 3 \\
PE as likely or more likely than an alternative diagnosis & 3 \\
Heart rate > I00 beats/minute & 1.5 \\
Immobilization or surgery in the previous 4 weeks & 1.5 \\
Hemoptysis & $\mathrm{I}$ \\
Malignancy & $\mathrm{I}$ \\
\hline Clinical probability (extended) & Total \\
Low & $0-1$ \\
Intermediate & $2-6$ \\
High & $\geq 7$ \\
Clinical probability (simplified) & \\
PE likely & $0-4$ \\
PE unlikely & $>4$ \\
\hline
\end{tabular}

Abbreviation: PE, pulmonary embolism. 
PPV 58\%) when clinical probability was inconsistent with the imaging result.

Recent studies suggest that MD-CTPA can reliably be used as the single imaging test for suspected PE in patients with low or intermediate clinical PE probability. ${ }^{18-22}$ The combination of clinical probability, D-dimer testing, and MD-CTPA proved to be safe for ruling out PE in several large prospective management studies. ${ }^{18-20}$ The 3-month risk for venous thromboembolic (VTE) events in patients who were left untreated after a negative MD-CTPA was $0.6 \%-1.5 \%{ }^{18-20}$ For comparison, conventional PA has a 3-month VTE risk rate of around $1 \%-2 \% .{ }^{23}$ Furthermore, the algorithm allowed a management decision in $98 \%$ of patients. ${ }^{19}$ As further evidence, two randomized trials suggested very low additional yield from confirmatory imaging of the leg veins in patients with normal MD-CTPA. ${ }^{21,22}$ The most recent study demonstrated that a strategy using D-dimer and MD-CTPA is equally safe as using D-dimer, venous ultrasonography of the leg, and MD-CTPA. ${ }^{22}$ The 3-month VTE rates were $0.3 \%$ in both groups.

False-negative MD-CTPA results are most often related to subsegmental thrombi. Indeed, the clinical relevance of isolated subsegmental thrombi is controversial. However, additional testing (eg, V/Q lung scanning) should be considered in patients with a high clinical probability of PE. ${ }^{14}$ False-positive CTPA results appear to be unusual. Limitations of CTPA include cost, relatively high radiation exposure, and contraindications to iodinated contrast material in patients with reduced renal function or iodine allergy. The radiation dose from MD-CTPA has been recently identified as an important public health problem especially in young women. ${ }^{24}$

The advantages of CTPA include speed, wide availability, adequate visualization of pulmonary arteries up to segmental and subsegmental vessels, definitive diagnosis of PE (either positive or negative), and the ability to establish alternative diagnoses. The disadvantages of CTPA include cost, high radiation exposure, and the possibility of inducing nephropathy or allergy associated with iodinated contrast material.

\section{Computed tomography venography}

The diagnostic value of additional computed tomography venography (CTV) in suspected PE was investigated in the PIOPED II study. Indeed, the combination of MD-CTPA and CTV revealed a higher sensitivity $(90 \%)$ for the diagnosis of PE than CTPA alone and a similar specificity ( $96 \%$ for CTPA alone and $95 \%$ for CTPA + CTV). However, the increase in NPV was less $(97 \%$ vs $96 \%) .{ }^{17}$ Since additional CTV increases radiation exposure without yielding significantly different predictive values compared with CT alone, this procedure is not recommended as routine diagnostic. ${ }^{25}$

The advantages are that combining CTPA with CTV increases sensitivity, but the disadvantage is that the absolute gain due to CTV is modest, and the overall radiation during examination is increased.

\section{V/Q scanning}

The basic principle for the diagnosis of PE based upon V/Q scanning is to recognize lung segments or subsegments without perfusion but preserved ventilation (called mismatch) (Figure 2).

In Germany, ventilation studies are usually carried out with radioaerosols or with Technegas (Cyclopharm, Melbourne, Australia). Technegas is an aerosol comprising Tc-99m-labeled carbon microparticles generated at high temperature, ${ }^{26,27}$ which have a diameter of about $0.005-$ $0.2 \mu \mathrm{m} .{ }^{28}$ The use of Tc-99m-Technegas has minimized the problem of hot spots in patients with obstructive lung disease and is according to clinical experience better than the
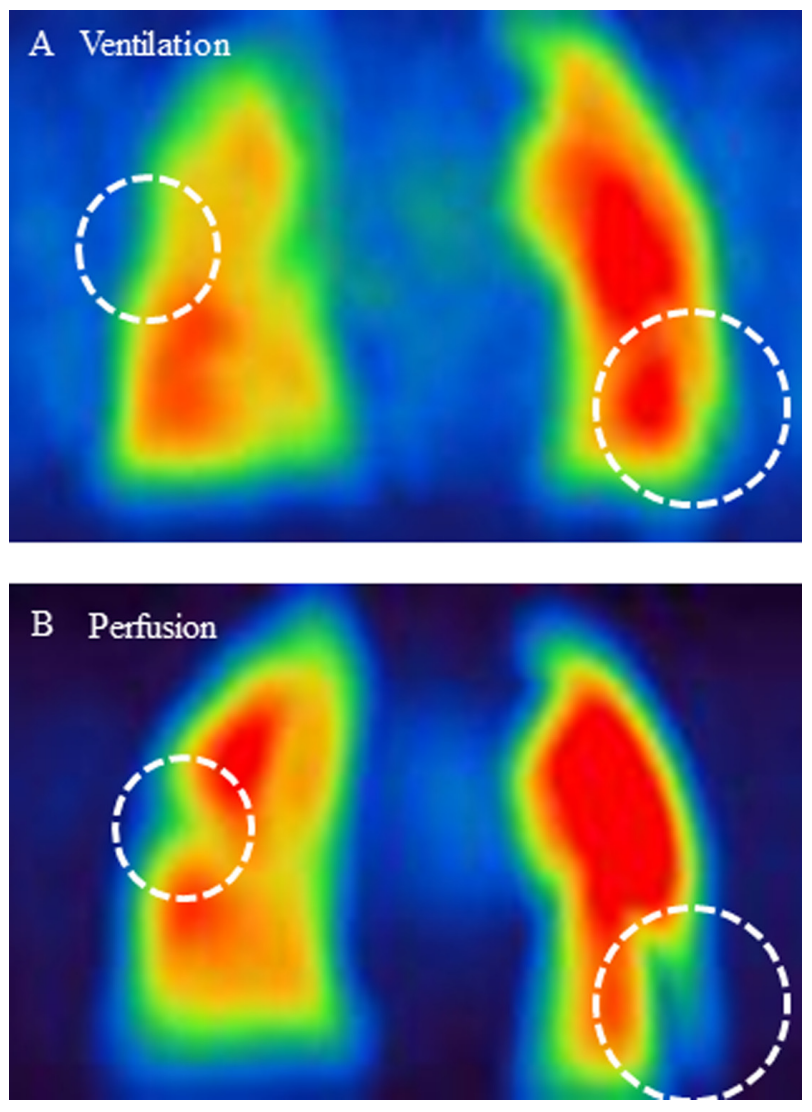

Figure 2 Diagnosis of pulmonary embolism with scintigraphy. A) Coronal ventilation and $\mathbf{B}$ ) coronal perfusion scintigraphy displays perfusion defects with intact ventilation (dotted circles) as a sign of pulmonary embolism. 
best liquid aerosols. Perfusion studies are performed after intravenous injection of macroaggregated human albumin (MAA).

\section{V/Q planar}

Since the publication of the first PIOPED study in 1990, many lessons have been learned concerning V/Q scanning. Detection of ventilation and perfusion defects at the subsegmental level is possible by planar imaging (V/Q planar) but is considerably better by V/Q-single photon emission computed tomography (SPECT). Poor results that were attributed to the performance of V/Q scanning can be in part explained by the use of planar images in the PIOPED studies. Follow-up studies provided a more detailed analysis on PIOPED data. ${ }^{29}$ More than 10 years after the publication of the first PIOPED study, V/Q scanning was validated in relation to a true gold standard using artificial subsegmental emboli. The sensitivity of V/Q planar was $67 \%$, while V/P-SPECT performed much better with $93 \%{ }^{30}$ In clinical studies, Bajc et al identified 53\% more mismatched regions with V/Q-SPECT than with V/Q planar. ${ }^{31}$

\section{V/Q-SPECT}

Nowadays, SPECT is the standard for how to perform V/Q studies (Figure 3), and SPECT-CT is the next diagnostic step. In addition to the above-mentioned study from Bajc et al, Collart et al showed that V/P-SPECT increased the specificity for PE from $78 \%$ to $96 \%$ at similar sensitivities. ${ }^{32}$ Reinartz et al found a sensitivity and specificity of $76 \%$ and $85 \%$, respectively, with V/Q planar compared with $97 \%$ and $91 \%$ with V/Q-SPECT..$^{33}$ In a head-to-head comparison, Gutte et al compared the performance of V/Q-SPECT with low-dose CT and MD-CT and found a sensitivity, specificity, and accuracy of $97 \%, 88 \%$, and $91 \%$, respectively, for V/Q-SPECT; $68 \%$, $100 \%$, and $88 \%$, respectively, for MD-CT, and $97 \%, 100 \%$, and $99 \%$, respectively, for V/Q-SPECT/low-dose CT. ${ }^{34}$

\section{Interpretation criteria for $\mathrm{V} / \mathrm{Q}$ scanning}

Probabilistic interpretation that was used in the PIOPED I and II studies ${ }^{17,35}$ were either never accepted in Germany or have been abandoned nowadays. An important step in improved reading of perfusion scans is the Prospective Investigative Study of Acute Pulmonary Embolism Diagnosis (PISA-PED) study. ${ }^{36}$ By using clinical evaluation and by recognition of perfusion patterns typical of $\mathrm{PE}$, the number of nondiagnostic examinations decreased significantly. According to contemporary understanding, holistic interpretation of scintigraphic images includes clinical information and laboratory test together with all observed signs and patterns on ventilation
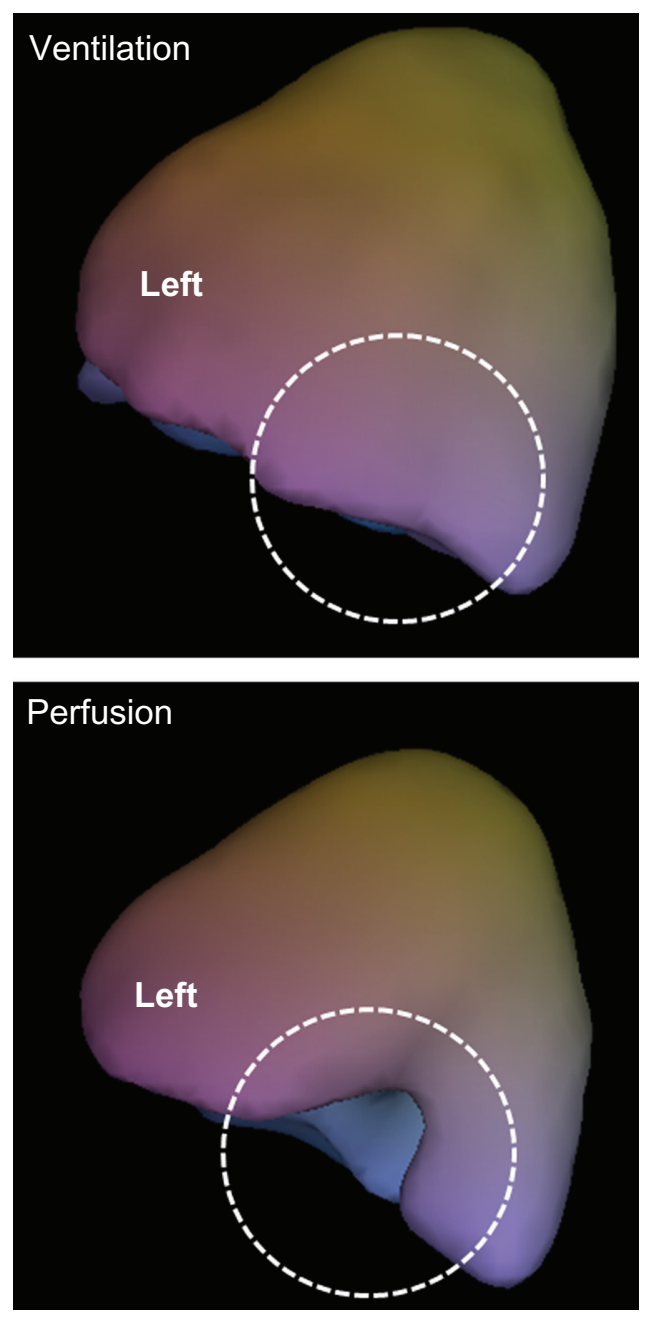

Figure 3 Diagnosis of PE with SPECT. 3D reconstruction of SPECT demonstrates a mismatch of ventilation and perfusion. The dotted circles mark parts of the left laterobasal pulmonary lobe where a wedge-shaped defect of the perfusion indicates a pulmonary embolism.

Abbreviations: 3D, three-dimensional; PE, pulmonary embolism; SPECT, single photon emission computed tomography.

and perfusion scintigrams. Schemes for clinical probabilities may be of significant value. ${ }^{37-39}$ Applying holistic principles of interpretation of V/P-SPECT, recent studies have shown NPVs in the range $97 \%-99 \%$, sensitivities in the range $96 \%-99 \%$, and specificities in the range $91 \%-98 \%$ for the diagnosis of PE. Rates of nondiagnostic findings were $1 \%-3 \%{ }^{33,40-42}$ Furthermore, the NPV of V/Q scanning for recurrent $\mathrm{PE}$ is unsurpassingly high.

Allergic reactions to Tc-99m-MAA used for lung perfusion imaging are very rare, and radiation exposure of $\mathrm{V} / \mathrm{Q}$ scanning is much lower in comparison to CT. According to the International Commission on Radiological Protection (ICRP), the effective radiation dose of $\mathrm{V} / \mathrm{Q}$ imaging is $1.8-2 \mathrm{mSv}^{43,44}$ Radiation exposure for the female breast is in the range of $0.8 \mathrm{mSv}^{43}$ and about a factor of 10 lower than CT. 
The advantages of $\mathrm{V} / \mathrm{Q}$ scanning are that it carries no contraindications and gives a low radiation burden. V/Q-SPECT has a high sensitivity and specificity for PE. However, the disadvantages are that V/Q planar findings are frequently inconclusive. V/Q-SPECT is in general less readily available.

\section{Pulmonary angiography}

For many decades, PA was regarded as the gold standard imaging technique for the diagnosis of PE with a sensitivity of around $98 \%$ and specificity between $95 \%$ and $98 \%{ }^{2,45}$ The two definitive diagnostic criteria include intraluminal filling defects and cutoff arteries as direct evidence of a thrombus (Figure 4). ${ }^{46}$ Other indirect signs such as hypoperfusion areas and asymmetric blood flow may be suggestive of PE but are not reliable when cardiorespiratory diseases coexist. PA is quite a safe examination, with mortality as low as $0.2 \%{ }^{47}$ However, this procedure has several limitations in clinical practice because it is invasive, expensive, and requires skillful physicians ${ }^{45,47}$ It is also often unavailable in smaller hospitals. Relative contraindications include significant bleeding risk and renal insufficiency. Notably, a negative PA result does not fully exclude VTE. The 3-month VTE rate after a normal PA has been reported to be $1.7 \% .{ }^{23}$ Furthermore, it has been suggested that PA has a limited interobserver agreement at subsegmental level (ranging between $45 \%$ and $66 \%$ ) and that sensitivity for subsegmental emboli may be suboptimal. ${ }^{48,49}$

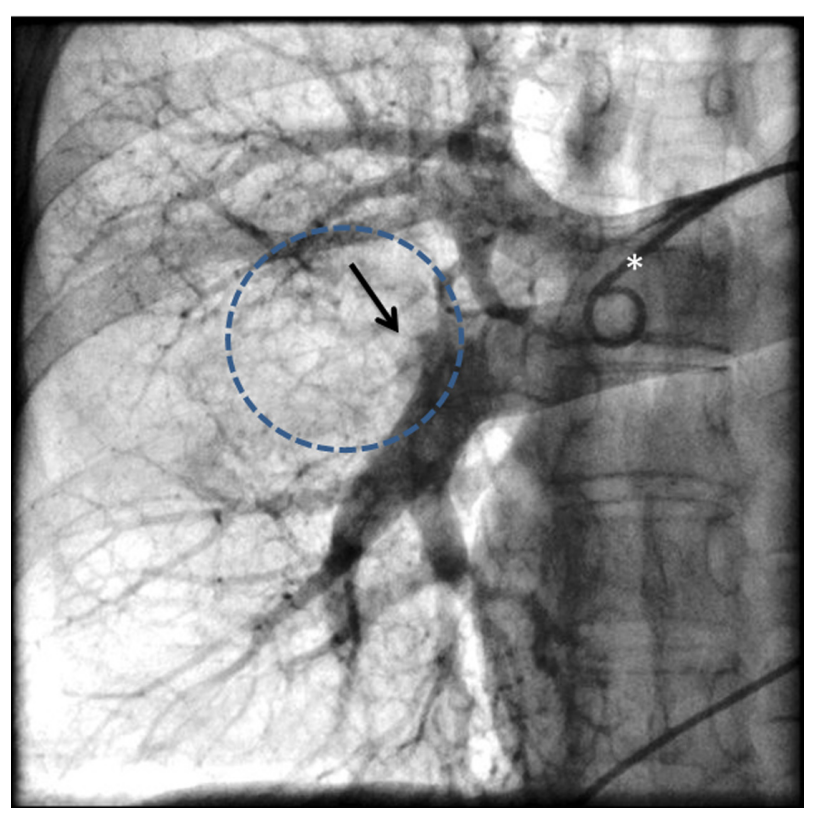

Figure 4 Diagnosis of pulmonary embolism with invasive angiography. Selective invasive right pulmonary artery angiogram demonstrates occlusion of a side branch of the right inferior pulmonary artery (arrow) with consecutive missing vessels in the marked area (dotted circle). Pigtail catheter is visualized in the right main artery (asterisk).
The use of conventional PA as an isolated diagnostic procedure has declined. PA has been abandoned in favor of noninvasive CTPA, which offers equivalent or even better information. Right ventriculography for the diagnosis of right ventricular failure from acute PE has been replaced by echocardiography and biomarkers. Furthermore, the risk of local bleeding complications increases if thrombolysis is attempted in patients in whom PE has been diagnosed by standard PA. ${ }^{50,51} \mathrm{PA}$ should be performed as the final diagnostic test whenever a diagnostic dilemma persists after noninvasive imaging tests. ${ }^{23}$ Advantages of PA include the option of direct hemodynamic measurements and catheterbased interventions, such as local thrombolysis, mechanical clot fragmentation, or catheter embolectomy.

The advantage of PA is its high diagnostic accuracy. It allows direct hemodynamic measurements and offers the option of catheter-based treatments. However, the disadvantage of PA is that it is invasive, costly, requires considerable expertise, and is not widely available. Contraindications include significant bleeding risk and renal insufficiency.

\section{Echocardiography}

The contribution of echocardiography to the diagnosis of PE is most often indirect because direct visualization of emboli within the right atrium, right ventricle (RV), or pulmonary artery occurs occasionally in only $4 \%$ of the patients with acute PE. ${ }^{52}$ Indirect echocardiographic signs of PE predominantly include signs of right ventricular overload or dysfunction (Table 2, Figure 5). ${ }^{53}$ However, in a prospective study including unselected patients suspected of having massive PE, transthoracic echocardiography failed to identify $50 \%$ of patients with angiographically proven PE. ${ }^{54}$ Furthermore, echocardiographic signs of right ventricular overload or dysfunction are not specific and might be due to pre-existing cardiorespiratory diseases. ${ }^{55}$ There is evidence suggesting that some echocardiographic findings may be more specific. The 60/60 sign (acceleration time of RV ejection $<60 \mathrm{~ms}$ and tricuspid insufficiency pressure gradient $\leq 60 \mathrm{~mm} \mathrm{Hg}$ )

Table 2 Potential echocardiographic findings in patients with pulmonary embolism. A definite discrimination between acute and pre-existing chronic changes is not possible

Right ventricular dilatation and hypokinesis (Figure 5B, D)

Paradoxical septal motion (Figure 5B)

Tricuspid regurgitation (Figure 5A)

Increased pulmonary artery pressure (Figure 5C)

Dilation of inferior vena cava without inspiratorical collapse

Dilation of proximal pulmonary arteries 

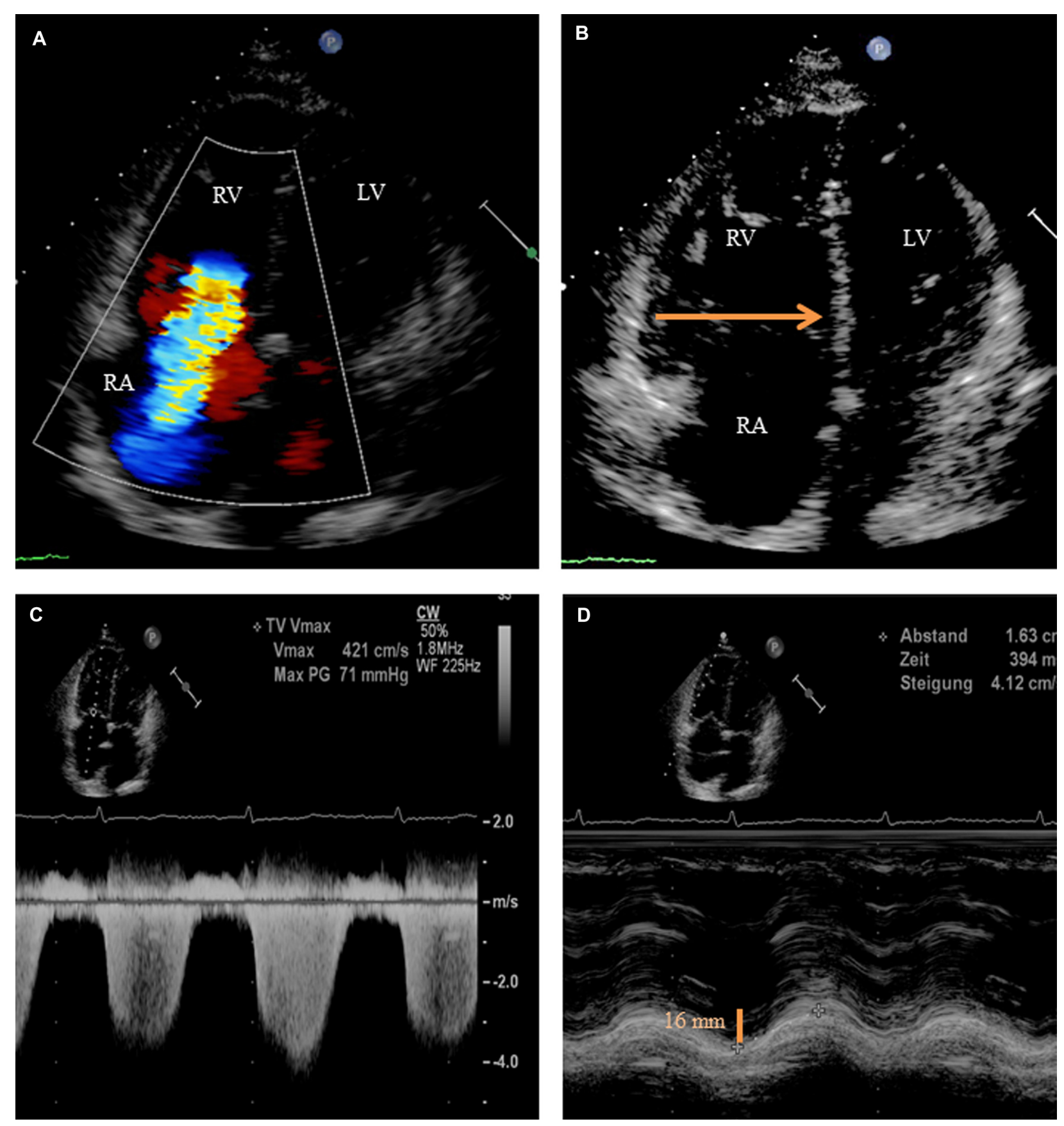

Figure 5 Echocardiography in PE. A) Color Doppler imaging reveals large tricuspid valve regurgitation. B) 2D imaging with enlarged right ventricle with deviation of the interventricular septum to left (arrow). C) Doppler echocardiography is used for the assessment of the acute elevated pulmonary artery pressure. D) Dysfunction of the right ventricle reflected by a reduced TAPSE.

Abbreviations: 2D, two-dimensional; PE, pulmonary embolism; TAPSE, tricuspid annular plane systolic excursion; RA, right atrium; RV, right ventricle; LV, left ventricle.

and the McConnell sign (normal apical segment of the right ventricular free wall in combination with akinesia of the right ventricular mid-free wall) have been reported to be insensitive (25\% and $19 \%)$, but highly specific (100\% and $94 \%$ ) for the diagnosis of acute PE ${ }^{56}$ Consequently, systematic use of echocardiography for diagnosis in hemodynamically stable, normotensive patients is not recommended in the current European Guidelines (evidence level IIIC)..$^{57,58}$

The main role of echocardiography in these patients is risk stratification since right ventricular dysfunction has been established as a powerful independent predictor of death from PE. ${ }^{52,59,60}$ Acutely unstable patients pose a different situation. 
In these patients, the absence of right ventricular overload or dysfunction practically excludes $\mathrm{PE}$ and may provide information that helps in the differential diagnosis of other causes of shock including acute left ventricular dysfunction, tamponade, acute valvular disease, and aortic dissection. ${ }^{61} \mathrm{On}$ the other hand, signs of right ventricular overload in a hemodynamically unstable patient with suspected PE are highly suggestive of PE and may justify thrombolysis if other diagnostic tests would result in additional risk or in delay of treatment. ${ }^{2}$ In support of this strategy, patients with a high clinical probability, a positive shock index ( $\geq 1$ ), and presence of right ventricular dysfunction underwent such a treatment with an acceptable all-cause mortality rate of $5 \%$ at 30 days. ${ }^{62}$

Because of the high prevalence of central PE in patients with hemodynamically significant PE, transesophageal echocardiography may allow direct visualization of a thrombus in the pulmonary artery and confirm the diagnosis in most cases. In a prospective study including 49 patients suspected of having massive PE with abnormal transthoracic echocardiograms, the sensitivity of transesophageal echocardiography for detecting PE of any size was $80 \%$ and its specificity was $100 \%{ }^{63}$

In hemodynamically unstable patients, the advantage of bedside echocardiography is that it is a valuable alternative if CT is not immediately available and may guide treatment or help in the differential diagnosis of the cause of the shock. However, in hemodynamically stable patients, the disadvantage of echocardiographic signs of PE are their nonspecificity which may be due to concomitant cardiorespiratory diseases.

\section{Diagnostic algorithm}

The diagnostic strategy depends on the severity of PE, which is understood as a PE-related early mortality risk and allows a distinction between high-risk PE presenting with shock or hypotension and nonhigh-risk PE without shock or hypotension. ${ }^{58}$ This classification guides the choice of the optimal initial management strategy with the purpose to avoid unnecessary radiological exposure without losing a high sensitivity to exclude clinically significant PE.

In suspected nonhigh-risk PE, the diagnostic workup should include the triage of clinical decision rule, D-dimer testing, and, if necessary, imaging. ${ }^{64,65}$ The most frequently used clinical prediction rule is the Wells score which is a simple rule based on easily collected information (Table 1). It has been validated extensively using both a three-category (low, moderate, or high clinical probability) and a two-category scheme (PE likely or unlikely). ${ }^{19,39,66-68}$

Several management studies have shown that PE can be ruled out without the need for further imaging in patients with low clinical probability and a normal D-dimer. ${ }^{19,22,69-73}$ Imaging is necessary in patients who either have an abnormal D-dimer result or have a high probability of PE irrespective of the D-dimer result. The first-line imaging modality is CTPA. CT-based algorithms have been well validated in prospective trials to safely diagnose or rule out PE. ${ }^{18-22}$ Clinicians should consider additional imaging whenever CTPA is inconsistent with the clinical probability. ${ }^{17}$ For patients with contraindication to CTPA, V/Q scan is a valid option. In the current guidelines for V/Q scintigraphy, V/Q-SPECT is recommended as the procedure of choice and is preferred to $\mathrm{V} / \mathrm{Q}$ planar (evidence level IIb). ${ }^{74}$ A normal V/Q-SPECT practically excludes PE, and positive findings lead to treatment in nearly all cases. Additional testing is required whenever lung scans are nondiagnostic. If the clinical suspicion of PE persists despite a negative V/Q planar result, the diagnosis should be rigorously pursued. ${ }^{74} \mathrm{PA}$ should be reserved for patients in whom a high clinical suspicion of $\mathrm{PE}$ persists despite a normal or noninvasive imaging. ${ }^{23}$

The diagnostic approach to suspected high-risk PE is different. Patients with high-risk PE presenting with hypotension or shock generally have a high clinical pretest probability. The most useful test in this situation is bedside transthoracic echocardiography, which will show indirect evidence of acute right ventricular overload or dysfunction if acute PE is the cause of hemodynamic condition. Rarely, right heart thrombi can be found on transthoracic echocardiography as a direct sign. ${ }^{52}$ In highly unstable patients or if other tests are not immediately available, the bedside echocardiographic findings alone may establish the diagnosis of PE. If the patient is stabilized, a definitive diagnosis should be sought by CTPA, which is usually able to confirm the diagnosis because of the high thrombus load in the pulmonary circulation.

In pregnant women with the suspicion of $\mathrm{PE}$, radiation exposure of the fetus is a concern. However, in most cases, the use of ionized radiation is indispensable as PE is a potentially fatal diagnosis. CTPA delivers a higher radiation dose to the mother, but a lower dose to the fetus than V/Q scanning. ${ }^{75,76}$ Thus, if necessary, CTPA is preferred during pregnancy in all trimesters. ${ }^{77}$

A straightforward diagnostic algorithm for suspected PE is presented in Figures 6 and 7. 


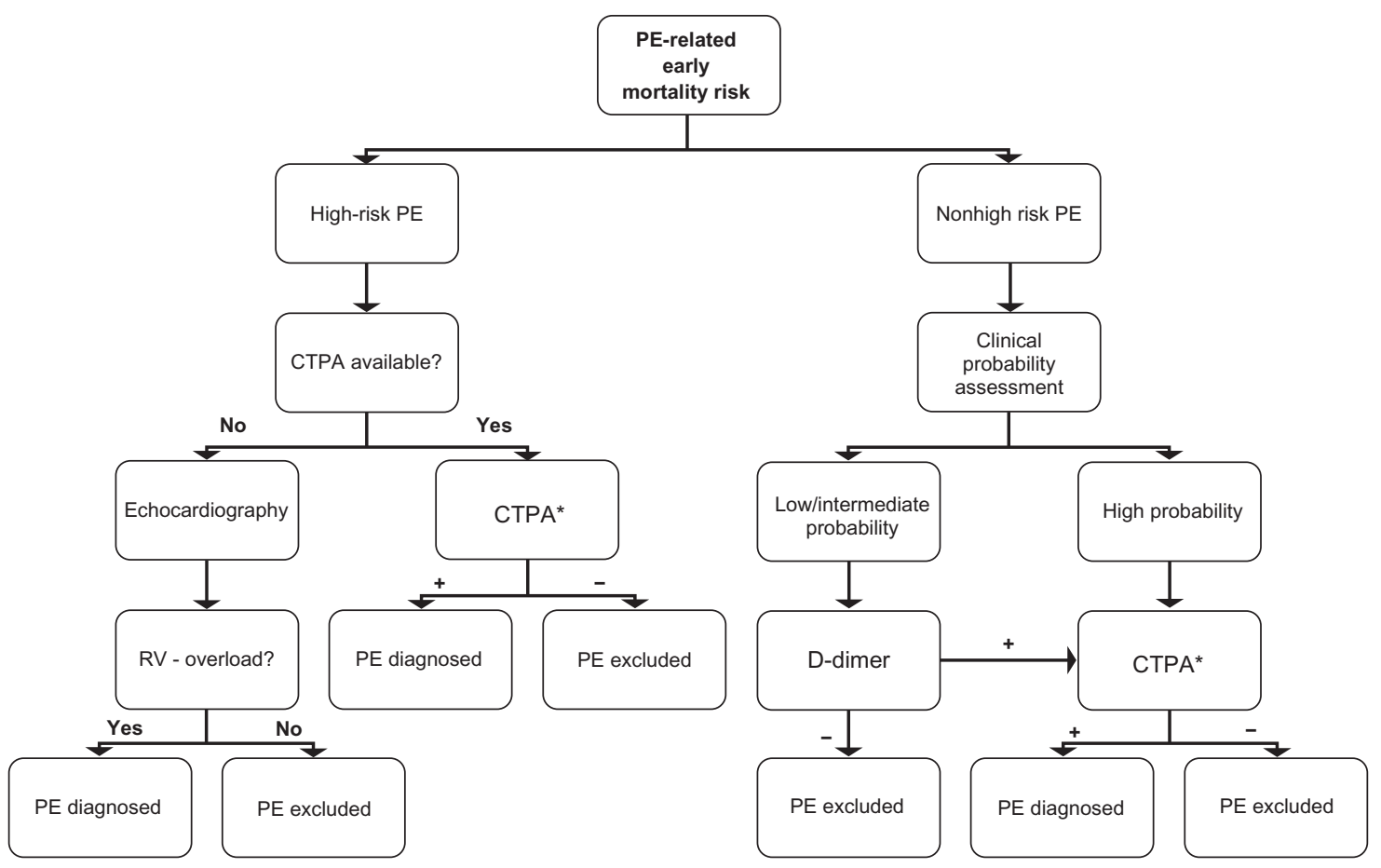

Figure 6 Algorithm in PE diagnosis. Risk stratification according to PE-related early mortality risk distinguishes high-risk PE (ie, presenting with shock or hypotension) and nonhigh-risk PE (ie, presenting without shock or hypotension). Clinical probability is most commonly assessed by Wells' criteria.

Note: 'When CTPA is contraindicated, V/Q scanning is an alternative.

Abbreviations: PE, pulmonary embolism; CTPA, computerized tomographic pulmonary angiography; RV, right ventricle; V/Q, ventilation-perfusion.

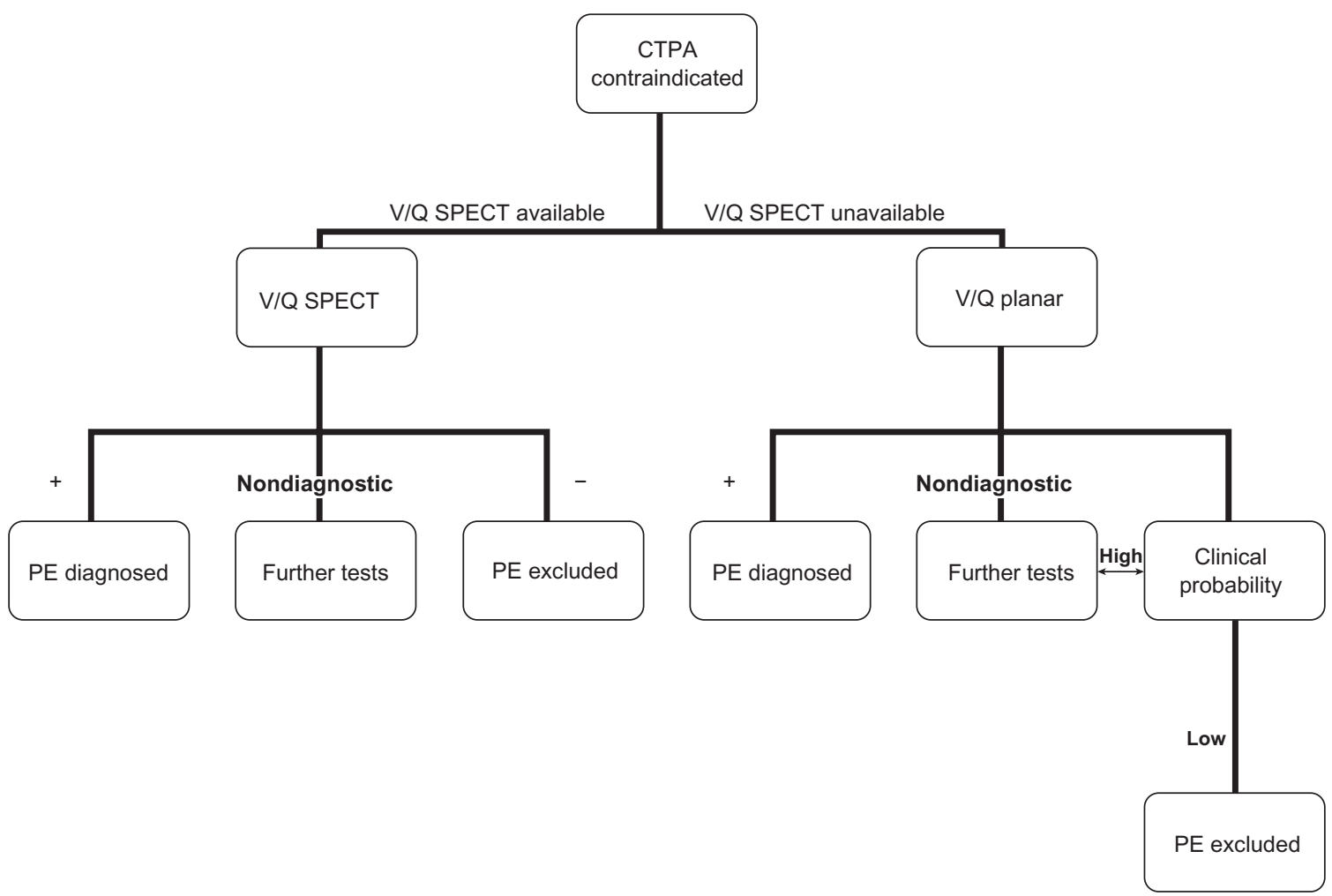

Figure 7 Algorithm for diagnostic imaging with V/Q scintigraphy. V/Q-SPECT is the procedure of choice. V/Q scans are interpreted according to the holistic principle in which clinical pretest probability is a part.

Abbreviations: V/Q-SPECT, ventilation-perfusion single photon emission computed tomography; V/Q planar, planar ventilation/perfusion; CTPA, computerized tomographic pulmonary angiography; $\mathrm{PE}$, pulmonary embolism. 


\section{Conclusion}

The routine application of imaging techniques is not warranted in patients with a low probability clinical assessment in combination with a negative $\mathrm{D}$-dimer result. When imaging is necessary, CTPA is the first-line test for investigating suspected PE. V/Q scintigraphy remains a validated option but is less frequently performed due to the high proportion of inconclusive results in planar V/Q scans. PA has been the traditional gold standard for the diagnosis of $\mathrm{PE}$, but it is now rarely performed. In skillful hands, PA can be used when confirmation is required after uncertain results of noninvasive imaging tests. Echocardiography has a limited clinical utility in the diagnosis of PE in hemodynamically stable, normotensive patients with suspected PE. Only in hemodynamically unstable patients is the detection or absence of echocardiographic signs of right ventricular dysfunction significant and justify initiation or withholding of thrombolytic therapy.

Despite the limited sensitivity and specificity of individual imaging techniques, their combination with the clinical assessment and D-dimer testing is a key step in all diagnostic algorithms and provides a useful strategy for risk stratification and optimal treatment in patients with suspected PE.

\section{Disclosure}

The authors report no conflicts of interest in this work.

\section{References}

1. Anderson FA Jr, Wheeler HB, Goldberg RJ, et al. A population-based perspective of the hospital incidence and case-fatality rates of deep vein thrombosis and pulmonary embolism. The Worcester DVT Study. Arch Intern Med. 1991;151(5):933-938.

2. Guidelines on diagnosis and management of acute pulmonary embolism. Task Force on Pulmonary Embolism, European Society of Cardiology. Eur Heart J. 2000;21(16):1301-1336.

3. Sandler DA, Martin JF. Autopsy proven pulmonary embolism in hospital patients: are we detecting enough deep vein thrombosis? J R Soc Med. 1989;82(4):203-205.

4. Bergqvist D, Lindblad B. A 30-year survey of pulmonary embolism verified at autopsy: an analysis of 1274 surgical patients. $\mathrm{Br} J$ Surg. 1985; 72(2):105-108.

5. Goldhaber SZ. Pulmonary embolism. N Engl J Med. 1998; 339(2):93-104.

6. Barritt DW, Jordan SC. Anticoagulant drugs in the treatment of pulmonary embolism. A controlled trial. Lancet. 1960;1(7138): 1309-1312.

7. Stein PD, Terrin ML, Hales CA, et al. Clinical, laboratory, roentgenographic, and electrocardiographic findings in patients with acute pulmonary embolism and no pre-existing cardiac or pulmonary disease. Chest. 1991;100(3):598-603

8. Sostman HD, Stein PD, Gottschalk A, Matta F, Hull R, Goodman L. Acute pulmonary embolism: sensitivity and specificity of ventilation-perfusion scintigraphy in PIOPED II study. Radiology. 2008;246(3):941-946.

9. Rathbun SW, Raskob GE, Whitsett TL. Sensitivity and specificity of helical computed tomography in the diagnosis of pulmonary embolism: a systematic review. Ann Intern Med. 2000;132(3):227-232.
10. Perrier A, Howarth N, Didier D, et al. Performance of helical computed tomography in unselected outpatients with suspected pulmonary embolism. Ann Intern Med. 2001;135(2):88-97.

11. Van Strijen MJ, De Monye W, Kieft GJ, Pattynama PM, Prins MH, Huisman MV. Accuracy of single-detector spiral CT in the diagnosis of pulmonary embolism: a prospective multicenter cohort study of consecutive patients with abnormal perfusion scintigraphy. J Thromb Haemost. 2005;3(1):17-25.

12. Musset D, Parent F, Meyer G, et al. Diagnostic strategy for patients with suspected pulmonary embolism: a prospective multicentre outcome study. Lancet. 2002;360(9349):1914-1920.

13. Perrier A, Roy PM, Aujesky D, et al. Diagnosing pulmonary embolism in outpatients with clinical assessment, D-dimer measurement, venous ultrasound, and helical computed tomography: a multicenter management study. Am J Med. 2004;116(5):291-299.

14. Tapson VF. Acute pulmonary embolism. N Engl J Med. 2008; 358(10):1037-1052.

15. Qanadli SD, Hajjam ME, Mesurolle B, et al. Pulmonary embolism detection: prospective evaluation of dual-section helical CT versus selective pulmonary arteriography in 157 patients. Radiology. 2000; 217(2):447-455.

16. Winer-Muram HT, Rydberg J, Johnson MS, et al. Suspected acute pulmonary embolism: evaluation with multi-detector row CT versus digital subtraction pulmonary arteriography. Radiology. 2004; 233(3):806-815.

17. Stein PD, Fowler SE, Goodman LR, et al. Multidetector computed tomography for acute pulmonary embolism. N Engl J Med. 2006; 354(22):2317-2327.

18. Perrier A, Roy PM, Sanchez O, et al. Multidetector-row computed tomography in suspected pulmonary embolism. N Engl J Med. 2005; 352(17):1760-1768.

19. van Belle A, Buller HR, Huisman MV, et al. Effectiveness of managing suspected pulmonary embolism using an algorithm combining clinical probability, D-dimer testing, and computed tomography. JAMA. 2006; 295(2):172-179.

20. Ghanima W, Almaas V, Aballi S, et al. Management of suspected pulmonary embolism (PE) by D-dimer and multi-slice computed tomography in outpatients: an outcome study. J Thromb Haemost. 2005;3(9):1926-1932.

21. Anderson DR, Kahn SR, Rodger MA, et al. Computed tomographic pulmonary angiography vs ventilation-perfusion lung scanning in patients with suspected pulmonary embolism: a randomized controlled trial. JAMA. 2007;298(23):2743-2753.

22. Righini M, Le Gal G, Aujesky D, et al. Diagnosis of pulmonary embolism by multidetector CT alone or combined with venous ultrasonography of the leg: a randomised non-inferiority trial. Lancet. 2008; 371(9621):1343-1352.

23. van Beek EJ, Brouwerst EM, Song B, Stein PD, Oudkerk M. Clinical validity of a normal pulmonary angiogram in patients with suspected pulmonary embolism-a critical review. Clin Radiol. 2001; 56(10):838-842.

24. Brenner DJ, Hall EJ. Computed tomography-an increasing source of radiation exposure. $N$ Engl J Med. 2007;357(22):2277-2284.

25. Konstantinides S. Clinical practice. Acute pulmonary embolism. NEngl J Med. 2008;359(26):2804-2813.

26. Burch WM, Tetley IJ, Gras JL. Technetium-99m 'pseudogas' for diagnostic studies in the lung. Clin Phys Physiol Meas. 1984;5(2):79-85.

27. Burch WM, Sullivan PJ, Lomas FE, Evans VA, McLaren CJ, Arnot RN. Lung ventilation studies with technetium-99m Pseudogas. J Nucl Med. 1986;27(6):842-846.

28. Lemb M, Oei TH, Eifert H, Günther B. Technegas: a study of particle structure, size and distribution. Eur J Nucl Med. 1993; 20(7):576-579.

29. Gray HW, Bessent RG. Pulmonary embolism exclusion: a practical approach to low probability using the PIOPED data. Prospective Investigation of Pulmonary Embolism Diagnosis. Eur J Nucl Med. 1998;25(3):271-276. 
30. Bajc M, Bitzén U, Olsson B, Perez de Sá V, Palmer J, Jonson B. Lung ventilation/perfusion SPECT in the artificially embolized pig. $J \mathrm{Nucl}$ Med. 2002;43(5):640-647.

31. Bajc M, Olsson CG, Olsson B, Palmer J, Jonson B. Diagnostic evaluation of planar and tomographic ventilation/perfusion lung images in patients with suspected pulmonary emboli. Clin Physiol Funct Imaging. 2004;24(5):249-256.

32. Collart JP, Roelants V, Vanpee D, et al. Is a lung perfusion scan obtained by using single photon emission computed tomography able to improve the radionuclide diagnosis of pulmonary embolism? Nucl Med Commun. 2002;23(11):1107-1113.

33. Reinartz P, Wildberger JE, Schaefer W, Nowak B, Mahnken AH, Buell U. Tomographic imaging in the diagnosis of pulmonary embolism: a comparison between V/Q lung scintigraphy in SPECT technique and multislice spiral CT. J Nucl Med. 2004;45(9):1501-1508.

34. Gutte H, Mortensen J, Jensen CV, et al. Detection of pulmonary embolism with combined ventilation-perfusion SPECT and low-dose CT: head-to-head comparison with multidetector CT angiography. J Nucl Med. 2009;50(12):1987-1992.

35. Value of the ventilation/perfusion scan in acute pulmonary embolism. Results of the prospective investigation of pulmonary embolism diagnosis (PIOPED). The PIOPED Investigators. JAMA. 1990; 263(20):2753-2759.

36. Miniati M, Pistolesi M, Marini C, et al. Value of perfusion lung scan in the diagnosis of pulmonary embolism: results of the Prospective Investigative Study of Acute Pulmonary Embolism Diagnosis (PISA-PED). Am J Respir Crit Care Med. 1996;154(5): 1387-1393.

37. Miniati M, Bottai M, Monti S, Salvadori M, Serasini L, Passera M. Simple and accurate prediction of the clinical probability of pulmonary embolism. Am J Respir Crit Care Med. 2008;178(3):290-294.

38. Perrier A. D-dimer for suspected pulmonary embolism: whom should we test? Chest. 2004;125(3):807-809.

39. Wells PS, Anderson DR, Rodger M, et al. Excluding pulmonary embolism at the bedside without diagnostic imaging: management of patients with suspected pulmonary embolism presenting to the emergency department by using a simple clinical model and d-dimer. Ann Intern Med. 2001;135(2):98-107.

40. Bajc M, Olsson B, Palmer J, Jonson B. Ventilation/perfusion SPECT for diagnostics of pulmonary embolism in clinical practice. $J$ Intern Med. 2008;264(4):379-387.

41. Leblanc M, Leveillée F, Turcotte E. Prospective evaluation of the negative predictive value of V/Q SPECT using $99 \mathrm{mTc}$-Technegas. Nucl Med Commun. 2007;28(8):667-672.

42. Lemb M, Pohlabeln H. Pulmonary thromboembolism: a retrospective study on the examination of 991 patients by ventilation/ perfusion SPECT using Technegas. Nuklearmedizin. 2001;40(6): $179-186$.

43. Radiation dose to patients from radiopharmaceuticals (addendum 2 to ICRP publication 53). Ann ICRP. 1998;28(3):1-126.

44. Radiological ICo. Icrp Publication 53: Radiation Dose to Patients from Radiopharmaceuticals. Amsterdam (The Netherlands): Elsevier Ltd; 1998.

45. Hudson ER, Smith TP, McDermott VG, et al. Pulmonary angiography performed with iopamidol: complications in 1,434 patients. Radiology. 1996;198(1):61-65.

46. Stein PD, O'Connor JF, Dalen JE, et al. The angiographic diagnosis of acute pulmonary embolism: evaluation of criteria. Am Heart $J$. 1967;73(6):730-741.

47. Stein PD, Athanasoulis C, Alavi A, et al. Complications and validity of pulmonary angiography in acute pulmonary embolism. Circulation. 1992;85(2):462-468.

48. Stein PD, Henry JW, Gottschalk A. Reassessment of pulmonary angiography for the diagnosis of pulmonary embolism: relation of interpreter agreement to the order of the involved pulmonary arterial branch. Radiology. 1999;210(3):689-691.
49. Diffin DC, Leyendecker JR, Johnson SP, Zucker RJ, Grebe PJ. Effect of anatomic distribution of pulmonary emboli on interobserver agreement in the interpretation of pulmonary angiography. AJR Am J Roentgenol. 1998;171(4):1085-1089.

50. Agnelli G, Becattini C, Kirschstein T. Thrombolysis vs heparin in the treatment of pulmonary embolism: a clinical outcome-based metaanalysis. Arch Intern Med. 2002;162(22):2537-2541.

51. Wan S, Quinlan DJ, Agnelli G, Eikelboom JW. Thrombolysis compared with heparin for the initial treatment of pulmonary embolism: a meta-analysis of the randomized controlled trials. Circulation. 2004; 110(6):744-749.

52. Goldhaber SZ, Visani L, De Rosa M. Acute pulmonary embolism: clinical outcomes in the International Cooperative Pulmonary Embolism Registry (ICOPER). Lancet. 1999;353(9162):1386-1389.

53. Goldhaber SZ. Echocardiography in the management of pulmonary embolism. Ann Intern Med. 2002;136(9):691-700.

54. Miniati M, Monti S, Pratali L, et al. Value of transthoracic echocardiography in the diagnosis of pulmonary embolism: results of a prospective study in unselected patients. Am J Med. 2001;110(7):528-535.

55. Bova C, Greco F, Misuraca G, et al. Diagnostic utility of echocardiography in patients with suspected pulmonary embolism. Am J Emerg Med. 2003;21(3):180-183.

56. Kurzyna M, Torbicki A, Pruszczyk P, et al. Disturbed right ventricular ejection pattern as a new Doppler echocardiographic sign of acute pulmonary embolism. Am J Cardiol. 2002;90(5):507-511.

57. Roy PM, Colombet I, Durieux P, Chatellier G, Sors H, Meyer G. Systematic review and meta-analysis of strategies for the diagnosis of suspected pulmonary embolism. BMJ. 2005;331(7511):259.

58. Torbicki A, Perrier A, Konstantinides S, et al. Guidelines on the diagnosis and management of acute pulmonary embolism: the Task Force for the Diagnosis and Management of Acute Pulmonary Embolism of the European Society of Cardiology (ESC). Eur Heart J. 2008; 29(18):2276-2315

59. Ribeiro A, Lindmarker P, Juhlin-Dannfelt A, Johnsson H, Jorfeldt L. Echocardiography Doppler in pulmonary embolism: right ventricular dysfunction as a predictor of mortality rate. Am Heart J. 1997; 134(3):479-487.

60. Grifoni S, Olivotto I, Cecchini P, et al. Short-term clinical outcome of patients with acute pulmonary embolism, normal blood pressure, and echocardiographic right ventricular dysfunction. Circulation. 2000; 101(24):2817-2822.

61. Arcasoy SM, Vachani A. Local and systemic thrombolytic therapy for acute venous thromboembolism. Clin Chest Med. 2003;24(1):73-91.

62. Kucher N, Luder CM, Dörnhofer T, Windecker S, Meier B, Hess OM. Novel management strategy for patients with suspected pulmonary embolism. Eur Heart J. 2003;24(4):366-376.

63. Pruszczyk P, Torbicki A, Pacho R, et al. Noninvasive diagnosis of suspected severe pulmonary embolism: transesophageal echocardiography vs spiral CT. Chest. 1997;112(3):722-728.

64. Le Gal G, Righini M, Parent F, van Strijen M, Couturaud F. Diagnosis and management of subsegmental pulmonary embolism. $J$ Thromb Haemost. 2006;4(4):724-731.

65. Wells PS, Ginsberg JS, Anderson DR, et al. Use of a clinical model for safe management of patients with suspected pulmonary embolism. Ann Intern Med. 1998;129(12):997-1005.

66. Anderson DR, Kovacs MJ, Dennie C, et al. Use of spiral computed tomography contrast angiography and ultrasonography to exclude the diagnosis of pulmonary embolism in the emergency department. JEmerg Med. 2005;29(4):399-404.

67. Sohne M, Kamphuisen PW, van Mierlo PJ, Buller HR. Diagnostic strategy using a modified clinical decision rule and D-dimer test to rule out pulmonary embolism in elderly in- and outpatients. Thromb Haemost. 2005;94(1):206-210.

68. Kearon C, Ginsberg JS, Douketis J, et al. An evaluation of D-dimer in the diagnosis of pulmonary embolism: a randomized trial. Ann Intern Med. 2006;144(11):812-821. 
69. Wells PS, Anderson DR, Rodger M, et al. Derivation of a simple clinical model to categorize patients probability of pulmonary embolism: increasing the models utility with the SimpliRED D-dimer. Thromb Haemost. 2000;83(3):416-420.

70. Kruip MJ, Slob MJ, Schijen JH, van der Heul C, Buller HR. Use of a clinical decision rule in combination with D-dimer concentration in diagnostic workup of patients with suspected pulmonary embolism: a prospective management study. Arch Intern Med. 2002;162(14):1631-1635.

71. Ten Wolde M, Hagen PJ, Macgillavry MR, et al. Non-invasive diagnostic work-up of patients with clinically suspected pulmonary embolism; results of a management study. J Thromb Haemost. 2004; 2(7):1110-1117.

72. Rodger MA, Bredeson CN, Jones G, et al. The bedside investigation of pulmonary embolism diagnosis study: a double-blind randomized controlled trial comparing combinations of 3 bedside tests vs ventilation-perfusion scan for the initial investigation of suspected pulmonary embolism. Arch Intern Med. 2006;166(2):181-187.
73. Goekoop RJ, Steeghs N, Niessen RW, et al. Simple and safe exclusion of pulmonary embolism in outpatients using quantitative D-dimer and Wells' simplified decision rule. Thromb Haemost. 2007;97(1): 146-150.

74. Bajc M, Neilly JB, Miniati M, Schuemichen C, Meignan M, Jonson B. EANM guidelines for ventilation/perfusion scintigraphy: Part 2. Algorithms and clinical considerations for diagnosis of pulmonary emboli with V/P(SPECT) and MDCT. Eur J Nucl Med Mol Imaging. 2009;36(9):1528-1538.

75. Marik PE, Plante LA. Venous thromboembolic disease and pregnancy. N Engl J Med. 2008;359(19):2025-2033.

76. Bourjeily G, Paidas M, Khalil H, Rosene-Montella K, Rodger M. Pulmonary embolism in pregnancy. Lancet. 2010;375(9713):500-512.

77. Winer-Muram HT, Boone JM, Brown HL, Jennings SG, Mabie WC, Lombardo GT. Pulmonary embolism in pregnant patients: fetal radiation dose with helical CT. Radiology. 2002;224(2):487-492.
Reports in Medical Imaging

\section{Publish your work in this journal}

Reports in Medical Imaging is an international, peer-reviewed, open access journal publishing original research, reports, reviews and commentaries on all areas of medical imaging. The manuscript management system is completely online and includes a very quick and fair peer-review system, which is all easy to use.

\section{Dovepress}

Visit http://www.dovepress.com/testimonials.php to read real quotes from published authors. 